\title{
GRB 100816A and the nature of intermediate duration gamma-ray bursts
}

\author{
Rachel L. Tunnicliffe and Andrew Levan \\ Department of Physics, University of Warwick, Coventry, CV4 7AL, UK \\ email: r.1.tunnicliffe@warwick.ac.uk
}

\begin{abstract}
Gamma-ray bursts are normally split into two classes, primarily determined by their observed duration, so called long $(>2 \mathrm{~s})$ and short $(<2 \mathrm{~s})$ GRBs. There have been many claims of a third duration class, with emission lasting for intermediate periods between $2-$ $5 \mathrm{~s}$, although the reality of this class remains controversial. Here, we investigate this further utilising the $2.9 \mathrm{~s}$ duration, spectrally hard GRB 100816A. This burst lies well offset from its host galaxy, has no evidence for an associated supernova (albeit to only moderately constraining limits), and has properties which appear to be genuinely intermediate between long- and shortpopulation bursts. We extend this analysis by comparing the physical locations of a population of intermediate duration GRBs with those of short-GRBs and long-GRBs, concluding that the intermediate sample is indistinguishable from the long-GRB population, whose locations are very different from other transients.
\end{abstract}

Keywords.

\section{Introduction}

Studies of BATSE and Swift GRBs have shown that their duration distribution can be best fit by the addition of a third class of burst, of intermediate duration, but typically softer spectra. In principle these GRBs could arise from progenitors distinct from those thought to form LGRBs and SGRBs, but they have proved extremely difficult to pinpoint (see e.g. de Ugarte Postigo et al. (2010)).

Here, we consider GRB 100816A, whose properties appear genuinely intermediate between LGRBs and SGRBs. The burst is formally long $(2.9 \mathrm{~s})$, but spectrally hard, and lies well offset from its host galaxy. No clear SN is detected to limits a factor of $\sim 10$ fainter than SN 1998bw.

\section{Properties of intermediate GRBs}

\subsection{Host galaxy properties}

The locations of transient objects are highly diagnostic of their progenitors. Fruchter et al. (2006) have shown that LGRBs are highly concentrated on their host light, much more so than core collapse supernovae generally. Further, Fong et al. (2009) have similarly shown that SGRBs are highly scattered on the light of their host galaxies. Using a sample of intermediate bursts figure 1(a) shows that the intermediate GRBs seem to trace the same locations as LGRBs, ruling out a location similar to SGRBs, or a linear proportionality to the light at the $98 \%$ and $90 \%$ confidence levels. This suggests that most intermediate GRBs are in fact arising from collapsars similar to LGRB progenitors.

We note that GRB 100816A itself does reside within a fainter region of its host but this is not unprecedented in the long GRB population. 

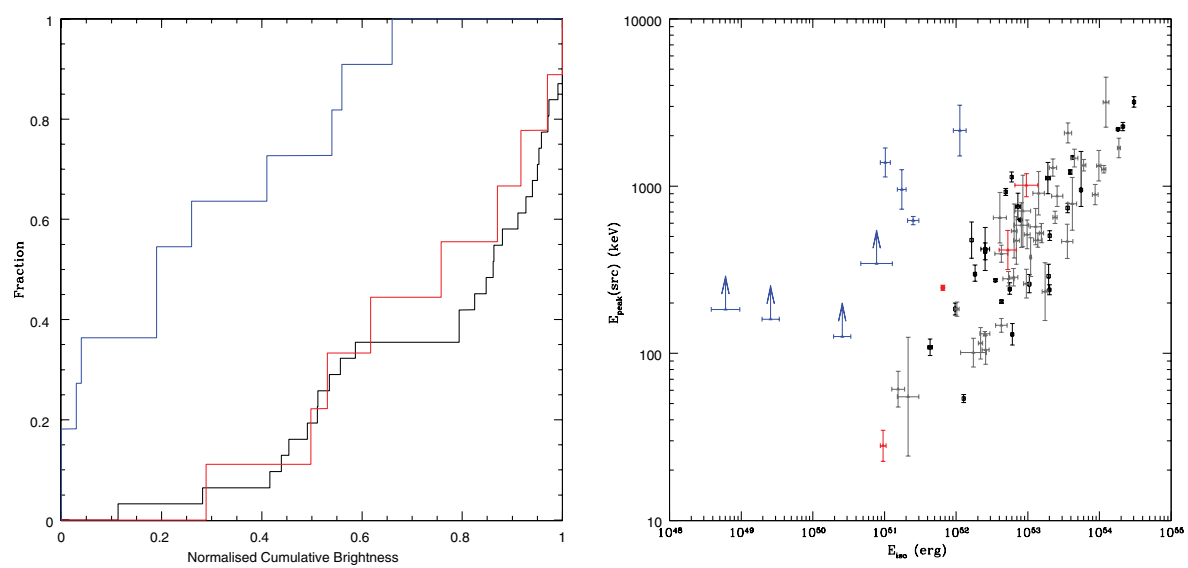

Figure 1. The left hand panel shows the distribution of short (blue), intermediate (red) and long (black) GRBs on the light of their host galaxies. Intermediate and long GRBs are highly concentrated on their host light and are clearly distinct from short GRBs. The right hand panel shows the energy characteristics of short (blue), intermediate (red) and long (black) GRBs in the rest frame of the burst. Long and intermediate GRBs follow the Amati relation whereas short GRBs clearly lie offset from this distribution.

\subsection{Prompt and afterglow characteristics}

Compared to other intermediate and long GRBs, GRB 100816A is spectrally hard. However, figure 1(b) shows that when looking at their intrinsic properties all intermediate GRBs, including GRB 100816A, follow the relation between the isotropic equivalent energy, $E_{i s o}$, and $E_{\text {peak }}$ in the rest frame of the GRB, first identfied by Amati et al. (2002). All short GRBs are well offset from this relation.

Comparing the optical afterglow magnitudes to values from Kann et al. (2010) and Kann et al. (2011) we find GRB 100816A to be intermediate between the long and short populations. For optical and X-ray afterglows de Ugarte Postigo et al. (2010) found this to be general property of intermediate GRBs.

\section{Conclusions}

We have shown that the majority of intermediate duration GRBs are distributed in host galaxies identically to the LGRBs, suggesting a very similar progenitor mechanism. However, some events such as GRB 100816A appear to lie in regions intermediate between LGRBs and SGRBs in many observational parameters. This suggests that, even with excellent observational data the task of distinguishing the origin of bursts around the $2 \mathrm{~s}$ divide is extremely challenging.

\section{References}

de Ugarte Postigo, A. et al. 2010, A\& A, 525, 109

Amati, L. et al. 2002, A\&A, 390, 81

Kann, D. A. et al. 2010, ApJ, 720, 1513

Kann, D. A. et al. 2011, ApJ, 734, 96

Fruchter, A. S. et al. 2006, Nature, 441, 463

Fong, W., Berger, E., \& Fox, D. B. 2009, ApJ, 708, 9 